\title{
Serumkomplementfaktoren, Akute Phase Proteine und zirkulierende Immunkomplexe bei Neurodermitikern vor und nach Testmahlzeiten*
}

\author{
G. Ionescu, D. Radovici, H. Mahal
}

Vital-Klinik, Alzenau-Michelbach

\begin{abstract}
Zusammenfassung:
Im Rahmen eines kontrollierten Testsystems (vor und nach standardisierten Testmahlzeiten) wurden bei 45 Neurodermitikern und 34 gesunden Kontrollpersonen die Werte der Serumkomplementfaktoren $C_{3 c}$, $C_{3}$-Aktivator und $C_{4}$, der Akuten Phase Proteine $\alpha_{1}$-Antitrypsin, $\alpha_{2}-$ Makroglobulin, Haptoglobin und Caeruloplasmin sowie der zirkulierenden Immunkomplexe (ZIK) mit IgE, IgG, IgM und IgA gemessen.
\end{abstract}

Signifikante Anstiege der $C_{3}$-Aktivator (Faktor B), der Akute Phase Proteine und der IgE-enthaltenden ZIK wurden bei einem erheblichen Teil der Neurodermitiker 15 Std. nach einer wiederholten Testmahlzeit registriert; dagegen blieben die. Werte bei den Kontrollpersonen in Normbereich.

Die Relationen zwischen den einzelnen Parametern sowie ihre diagnostisch-klinische Relevanz bei Atopikern nach Gabe definierter Testmahlzeiten wird diskutiert.

\section{Schlüsselwörter:}

Atopische Dermatitis - Komplementfaktoren - Akute Phase Proteine - zirkulierende Immunkomplexe Testmahlzeit

\section{Summary:}

The serum complement factors $C_{3 c}, C_{3}$-activator and $C_{4}$, the acute phase proteins $\alpha_{1}$-antitrypsin, $\alpha_{2}$-macroglobulin, haptoglobin and ceruloplasmin as well as the circulating immune complexes (CIC) with IgE, IgG, IgM and IgA were investigated in 45 atopic dermatitis (acute dermatitis) patients and 34 healthy controls before and after challenge meals (CM). Significantly increased serum levels of $C_{3}$-activator (Factor $B$ ), acute phase proteins and IgE-containing CIC were registered $15 \mathrm{~h}$ after $C M$ in the atopic dermatitis group; by contrast all values remained in the normal range in the control group.

The relations between single parameters as well as their diagnostic/clinical significance in atopic patients after CM administrations are discussed.

Keywords:

atopic dermatitis - complement factors - acute phase proteins - circulating immune complexes challenge meal---

\section{Einleitung}

Die Bedeutung der Nahrungsallergene in der Pathogenese der atopischen Dermatitis (Neurodermitis) ist umstritten. Einerseits hat sich keine Korrelation zwischen den Ergebnissen der Hauttests und der klinischen Symptome nach Provokationsverfahren erwiesen (1-3), und andererseits liegen bisher über die Anwesenheit und die klinische Relevanz der spezifischen IgE-Antikörper oder der zirkulierenden Immunkomplexe nach Nahrungseinnahme nur sporadische Studien vor (4-7). Die Relationen zwischen den 0.9 . Parametern einerseits und weiterer pathogenetischer Aspekte der akuten Dermatitis, wie Histaminfreisetzungen oder Darmpermeabilitätsstörungen andererseits wurden von uns in einer früheren Studie untersucht (8).

\footnotetext{
- Nach einem Vortrag anläßlich der Dermatologischen Gemeinschaftstagung in Minden, 18.-20. Oktober 1985
}

Der jetzige Bericht versucht im Rahmen eines kontrollierten Verfahrens vor und nach standardisierten Testmahlzeiten, die Rolle der Nahrung bei der Auslösung pathogener immunbiologischer Reaktiōnen bei Atopikern weiterhin zu dokumentieren. Die Abweichungen der Serumkomplementfaktoren, der Akute Phase Proteine und der zirkulierenden Immunkomplexe verschiedener Ig-Klassen dienten als Maßstab der Nahrungseinwirkung.

\section{Materialien und Methoden}

\section{Patienten und Kontrollkollektiv}

Insgesamt 45 Patienten mit gesicherter atopischer Dermatitis-Diagnose (9) im Alter von 15-48 Jahren und 34 gesunde Kontrollpersonen (18-50 Jahre) nahmen an den beschriebenen immunbiologischen Untersuchungen teil. Erhöhte Eosinophilenzahlen und Gesamt-Serum- 
IgE-Werte wurden bei allen 45 akuten Dermatitis-Patienten registriert, wobei in 33 Fällen Werte über $1000 \mathrm{U} / \mathrm{ml}$ gemessen wurden. Keiner der Patienten zeigte bei der Aufnahme asthmatische Symptome, und alle waren schon seit mindestens 10 Tagen vor Versuchsbeginn steroid- und antihistaminikafrei. In 44 atopischen Dermatitis-Fällen wurde von der Hautoberfläche hämolysierender Staph. aureus isoliert, und 29 Patienten wiesen weitere mucokutane Infektionen mit Streptococcus pyogenes, Candida sp., Aspergillus sp., Enterokokken und/oder Herpes simplex Virus auf.

Sowohl bei den Patienten als auch bei den Kontrollpersonen erfolgten Blutabnahmen vor und 2 bzw. 15 Std. nach einer (wiederholten) Testmahlzeit. Die Testmahlzeiten bestanden aus exakt abgemessenen Mengen von verschiedenen üblichen Nahrungsmitteln' mit einer Antigenstruktur entsprechend den 25 getesteten RAST-Nahrungsallergenen (8). Aufgrund früherer Reduktionsdiätbzw. Provokationsverfahren waren sich 20 von 45 Neurodermitikern positiver Reaktionen auf 1, 2 oder 3 Nahrungsmittel bewußt.

\section{Immunbiologische Untersuchungen}

Die Bestimmung zirkulierender Immunkomplexe (ZIK) verschiedener Ig-Klassen vor und 15 Std. nach einer Testmahlzeit erfolgte bei 35 Atopikern und 20 Kontrollpersonen mittels einer PEG-lasernephelometrischen Technik (10), wobei als positive Resultate nur die erhöhten ZIK-Werte ( $>\bar{x}+2$ SD) registriert wurden. Die Messung der IgG-, IgM-, IgA- und IgE-Komponenten in den aufgelösten PEG-Präzipitaten wurde mit Hilfe spezifi-

1 Die genaue Zusammensetzung der Testmahlzeit stellt die VitalKlinik auf Anfrage zur Verfügung scher Antisera (Behringwerke, Marburg) bzw. mit Phadebas-PRIST für IgE (Pharmacia, Freiburg) durchgeführt.

Als Vertrauensbereich für die ZIK-Werte $(\bar{x}+2$ SD) dienten uns die Ergebnisse eines Kontrollkollektivs von 220 gesunden Personen, die dem Institut für klinische Immunologie und Rheumatologie der Universität Erlangen/ Nürnberg bereits vorlagen.

Die Serumkomplementfaktoren $C_{3 c}, C_{3}$-Aktivator (Faktor $B$ ) und $C_{4}$ sowie die akute phase Proteine $\sigma_{1}$-Antitrypsin, $\alpha_{2}$-Makroglobulin, Haptoglobin und Caeruloplasmin wurden bei 10 Neurodermitikern und 8 Kontrollpersonen vor und 2, bzw. 15 Std. nach einer wiederholten allergenreichen Testmahlzeit mit einer radialen ImmundiffusionsMethode (M-Partigen, Behringwerke, Marburg) untersucht (11). Die Normwerte.(in $\mathrm{mg} / \mathrm{dl}$ ) sind in Abb. 2 und 3 dargestellt.

Die getrennten Serumproben für die o.g. Tests wurden je nach Zeit der Blutabnahme numeriert, binnen 3 Std. bei $-25^{\circ} \mathrm{C}$ gelagert und durch ein Blindverfahren am Institut für Immunologie und Rheumatologie der Universität Erlangen/Nürnberg untersucht.

Die Ergebnisse wurden statistisch ausgewertet im Student-t-Test.

\section{Resultate und Diskussion}

\section{Zirkulierende Immunkomplexe}

Erhöhte Serum-Werte der ZIK ( $>\bar{x}+2$ SD) mit IgE wurden in 16 von 34 Fällen (47\%), mit IgG in 12 von 35 Fällen $(34,3 \%)$, mit IgM in 19 von 35 Fällen. $(54,3 \%)$ und

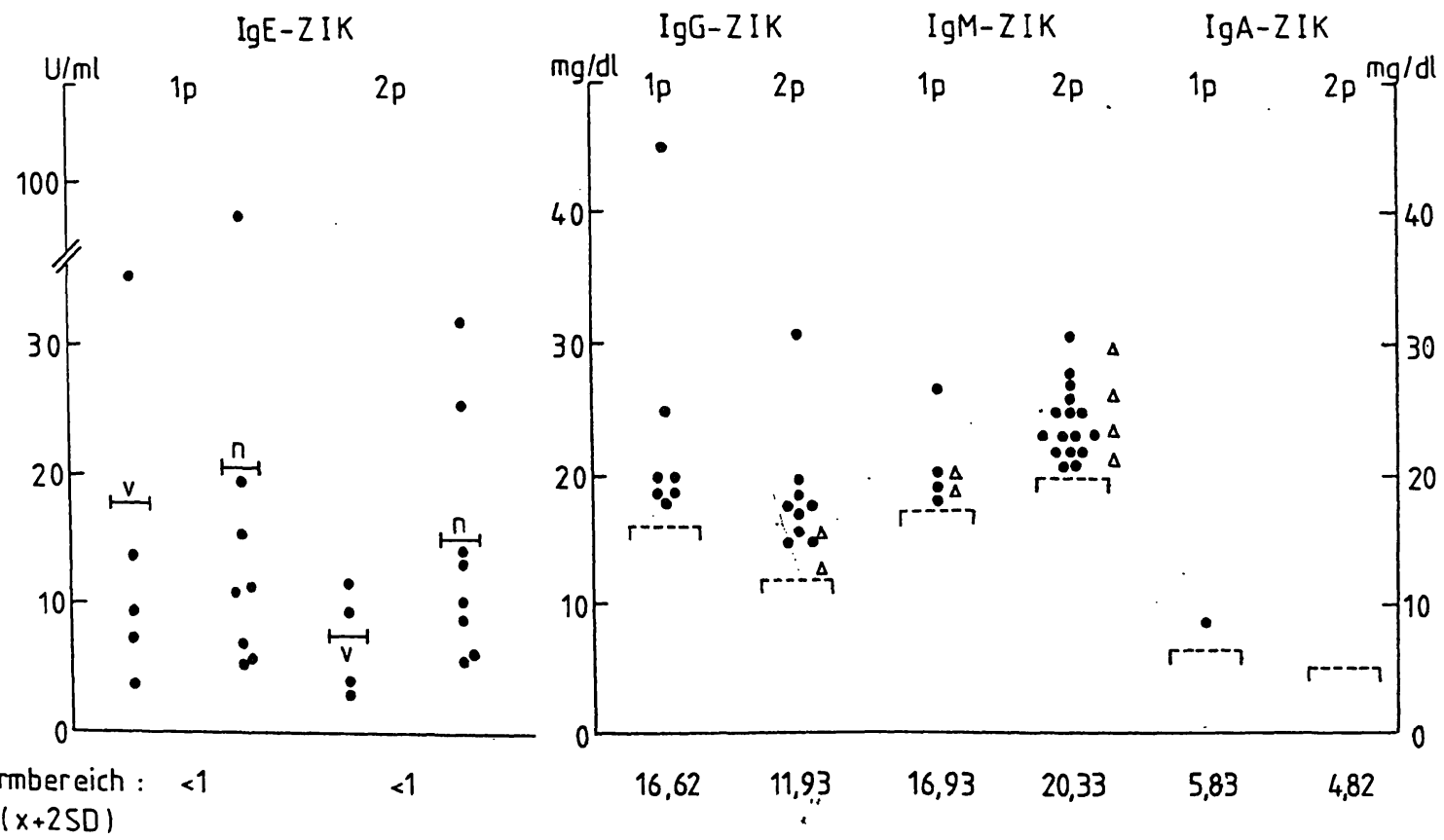

Abb. 1: Positive Serumwerte der zirkulierenden Immunkomplexe (ZIK) bei 35 akuten Dermatitis-Patienten und 20 Kontrollpersonen 15 Std. nach einer Testmahlzeit

O: Positive ZIK-Werte der 35 akuten Dermatitis-Patienten. $\triangle$ : Positive ZIK-Werte der 20 Kontrollpersonen. — : IgE-ZIK-Mittelwerte. $v$ : vor Testmahlzeit, $n$ : nach Testmahlzeit. _- : Normbereich $(x+2$ SD) eines Kontrollkollektivs von 220 gesunden Personen. 1 p, 2 p: erstes, bzw. zweites Präzipitat der-PEG-Lasernephelometrischen Methode 
mit $\lg A$ in 1 von 35 Fällen (2,85\%) gemessen. Dagegen zeigten sich nur bei 2 bzw. 4 der 20 getesteten Kontrollpersonen positive Serumwerte der IgG- bzw. IgM-ZIK nach Testmahlzeit (Abb.1). Die errechneten IgE-ZIKMittelwerte zeigten eine deutliche Steigerung nach Testmahlzeit, v. a. im 2. Präzipitat (Abb. 1); eine Tatsache, die an eine Komplexierung der Nahrungsallergene denken läßt. Gleichzeitig wiesen 16 von 20 akuten DermatitisPatienten mit bekannten allergischen Reaktionen gegen bestimmte Nahrungsmittel IgE-ZIK im Serum auf (Korrelationsmaß 80\%).

Zwischen den Gesamt-Serum IgE-Werten einerseits und der Häufigkeit positiver RAST- bzw. IgE-ZIK Resultate andererseits wurde in derselben atopischen DermatitisGruppe eine direkte Korrelation nachgewiesen (8). Die häufig beobachteten Schwankungen dieser Komponente nach oben oder unten kann man als ein dynamisches Gleichgewicht, bestehend aus Reaktionssynthese des spezifischen IgE, Komplexbildung zwischen spezifischem IgE und den entsprechenden Antigen (6), Abbau solcher Komplexe durch Antigen-Überschuß oder durch Abfangen im RES interpretieren.

Die anderen Ig-enthaltenden ZIK wiesen keine wesentlichen Abweichungen 15 Std. nach Testmahlzeit auf; ihre konstant erhöhten Mittelwerte und Häufigkeit in der akuten Dermatitis-Gruppe (insbesonders IgG-ZIK im 1. Präzipitat) deuten unserer Meinung nach vielmehr auf eine Beteiligung dieser Ig-Klassen an einem chronischen $\mathrm{Ab}$ wehrprozeß hin, die in direkter Relation mit den allgemein beobachteten Hautinfektionen stehen kann. Es ist jedoch nicht auszuschließen, daß solche Komplexe auch Nahrungsantigene enthalten (7).
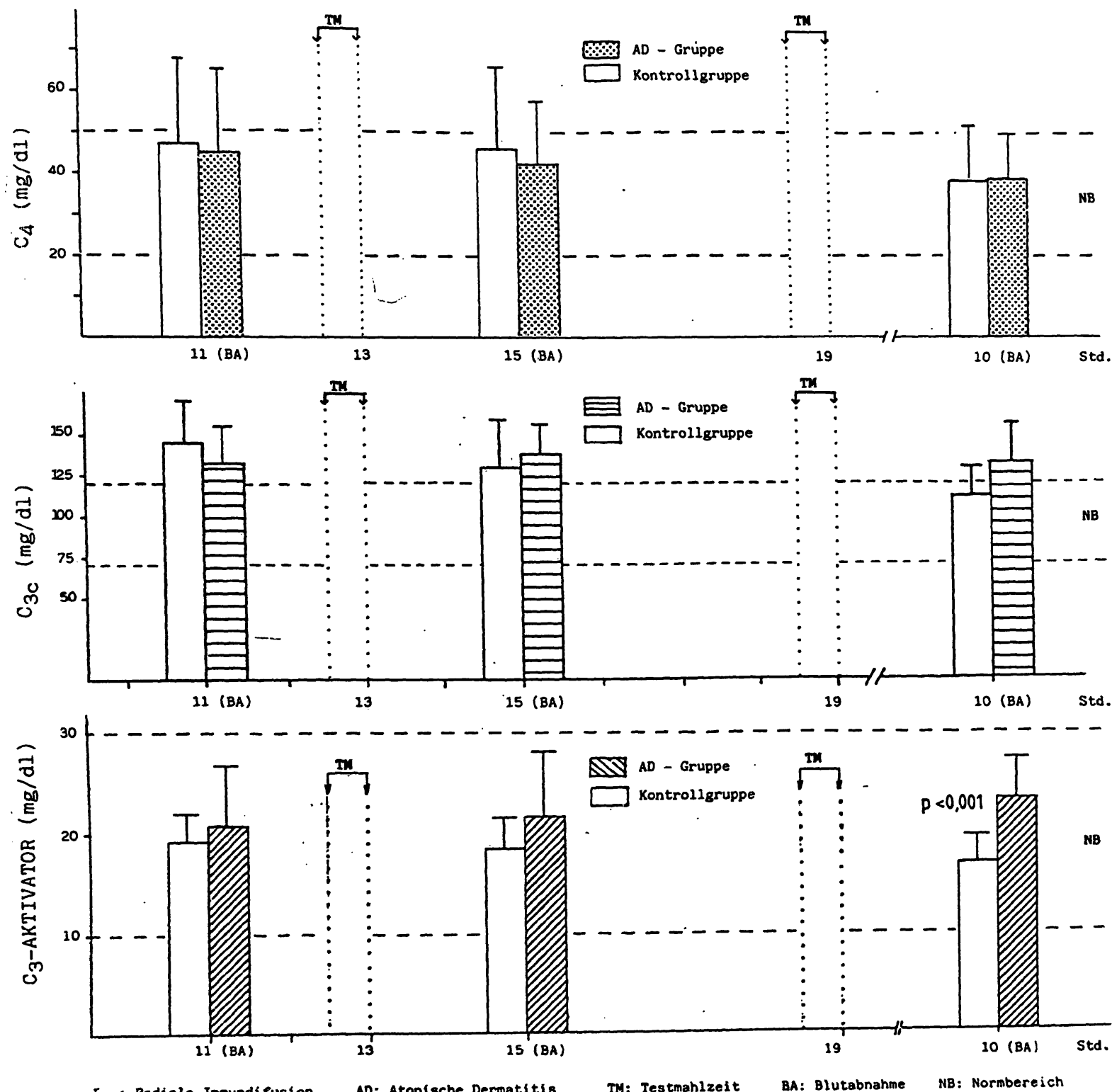

Abb.2: RID-Bestimmung der Serum-Komplementfaktoren $C_{4}, C_{3 c}$ und $C_{3}$-Aktivator (Faktor B) bei Neurodermitikern und Kontrollpersonen vor und nach standardisierten Testmahlzeiten 
Eine gesteigerte Nahrungsmittelantigenabsorption auf dem Darmniveau $(8,12)$, kombiniert mit einer permanen. ten Freisetzung von mikrobiellen Antigenen bzw. Toxinen bieten unserer Meinung nach die besten Voraussetzungen für die Uberbelastung des RES und für die Aufrechterhaltung erhöhter IgE-, IgG- und IgM-ZIK-Werte bei Neurodermitis-Patienten.

Daß zirkulierende Immunkomplexe zu einer Aktivierung des Komplementsystems $(13-15)$ und des Gerinnungsprozesses $(16,17)$ oder zur Hemmung der zellulären Immunfunktion $(18,19)$ beitragen können, ist schon bekannt, und solche Nebenwirkungen wurden auch bei akuten Dermatitis-Patienten beobachtet $(6,20)$.

Die erwiesene Korrelation (80\%) zwischen der Anwesenheit der IgE-ZIK und dem Auftreten klinischer Symptome gegen Nahrungsmittel bei unseren Neurodermitikern untermauert diese Aussage.

\section{Serum-Komplementfaktoren}

Leichte Abweichungen nach unten zeigten die Mittelwerte des Komplementfaktors $\mathrm{C}_{4}$ der beiden Gruppen (akute Dermatitis und Kontrollpersonen) 2 bzw. 15 Std. nach einer wiederholten Testmahlzeit (Abb.2). Sie blieben aber ständig im Normbereich.

Ebenfalls ohne signifikante Unterschiede zwischen den beiden Gruppen erfolgte die Untersuchung der $\mathrm{C}_{3 c}$-Fraktion. Dies blieb aber stets über der Normgrenze in der akuten Dermatitis-Gruppe (MW zwischen 129,4 und $159,1 \mathrm{mg} / \mathrm{dl}$ ). Ähnliche Anstiege der $\mathrm{C}_{3}$-Fraktion des Komplementsystems in Seren von Neurodermitikern wurden auch von anderen Autoren beobachtet und auf die Anwesenheit sekundärer Infektionen zurückgeführt (21).

Die $\mathrm{C}_{3}$-Aktivator-Fraktion bzw. Faktor B wies von Anfang an bei Neurodermitikern höhere Werte als bei den Kontrollpersonen auf (Abb.2). Nach der 2. Testmahlzeit wurde die Differenz signifikant: $23,6 \pm 3,2$ vs 16,6 $\pm 2,5 \mathrm{mg} / \mathrm{dl}(\mathrm{p}<0,001)$.

Eine mögliche Aktivierung des alternativen Weges des Komplementsystems durch IgE-ZIK (13) - siehe die gleichzeitig erhöhten IgE-ZIK-Werte 15 Std. nach Testmahlzeit - verbunden mit der Aufteilung des Faktor $B$ in seine $B_{a}$ - und $B_{b}$-Spaltprodukte könnte die signifikante Erhöhung dieser Fraktion nach Testmahlzeit erklären.

Da die hier angewandte radiale Immundiffusions-Methode nur wenige Auskünfte über die Funktion des Komplementsystems anbieten kann, wird z. Zt. im o.g. Testsystem die Untersuchung hämolytischer Aktivitäten verschiedener Komplementfaktoren vorbereitet. In einer früheren Studie wurde schon eine merkliche Aktivitätsabnahme der $\mathrm{CH}_{50}$ in Seren von 2 Atopikern nach Nahrungsmittel challenge demonstriert (6).

\section{Akute Phase Proteine}

Die Ausgangswerte der Akute Phase Proteine $\alpha_{1}$-AT, $\alpha_{2}-M$, Haptoglobin und Caeruloplasmin lagen schon höher bei Neurodermitikern als bei Kontrollpersonen (Abb. 3). Zwei Stunden nach der 1. allergenreichen Testmahlzeit wurden die Unterschiede signifikant bei $\alpha_{1}$-AT, Haptoglobin und Caeruloplasmin ( $p<0,05$ ); 15 Std. nach der 2 . Testmahlzeit wurden weitere Anstiege registriert bei $\alpha_{1}$-AT ( $\left.p<0,001\right), \alpha_{2}$-Makroglobulin ( $p<$ $0,02)$ und Caeruloplasmin $(p<0,02)$, die jetzt gut über der Normgrenze lagen (Abb. 3). Dagegen wiesen alle o.g. Serumproteine in der Kontrollpersonen-Gruppe weder si- gnifikante Abweichungen nach Testmahlzeit noch Werte außerhalb des Normbereiches auf.

Die signifikanten Anstiege der ${\mu_{1}}_{1}$-AT und $\pi_{2}-M$ darf man hier als eine antiplasminische Aktivitätsreaktion interpretieren, die im Verlauf eines erhöhten Gerinnungs- bzw. fibrinolytischen Prozesses auftreten kann (22). Daß zirkulierende Immunkomplexe zu einer Aktivierung des $\mathrm{Ge}$ rinnungsprozesses beitragen können, ist schon bekannt $(16,17)$, und dies wurde auch bei unseren akuten Dermatitis-Patienten beobachtet (Thrombozytenaggregationsfähigkeit, clotting time, Antithrombin III, FSP - unveröffentlichte Ergebnisse).

Die kompensatorische Erhöhung der zwei Fibrinolysehemmer $\left(\alpha_{1}-\right.$ AT und $\left.\alpha_{2}-M\right)$ kann allerdings auch unerwünschte Nebenwirkungen mit sich bringen:

- Einerseits eine Inhibition verschiedener Verdauungsenzyme (Trypsin, Chymotrypsin, Pepsin), was seinerseits zur Absorption unvollständig abgebauter Proteinspaltprodukte mit einem erhöhten allergischen Potential führen kann, und

- andererseits einen relativen Anstieg der Blutviskosität durch erhöhte $\alpha_{2}$-Makroglobulin-Werte, was die Erythrozyten-Geldrollen-Formation in den kleinen Blutgefäßen begünstigt und zur peripheren Minderdurchblutungen beitragen kann (23).

Der Anstieg des Haptoglobinwertes dürfte aufgrund der peroxidaseähnlichen Aktivität des Haptoglobin- $\mathrm{Hb}$ Komplexes (22) als ein protektiver Mechanismus gegen die von Leukozyten freigesetzten $\mathrm{H}_{2} \mathrm{O}_{2}$ und andere freie Radikale angesehen werden. Das kann im Darm nach Testmahlzeit (24) oder in den geschädigten Hautarealen erfolgen. Ein beschleunigter Hämoglobin turn-over be unseren superinfizierten akuten Dermatitis-Patienten konnte auch zu einer reaktiven Erhöhung des Haptoglobins beitragen.

Schließlich kann man den Caeruloplasmin-Anstieg als eine protektive Antioxidant-Aktivität gegen beschädigte Lipoproteinstrukturen im Darm oder in der Haut ansehen, wie bereits bei anderen entzündlichen Prozessen (25) bekannt ist. Die von uns beobachteten schnellen Caeruloplasmin-Schwankungen nach oben (schon $1 / 2$ Std. nach Testmahlzeit) dürften gleichzeitig auf seine Phenoloxidase-Aktivität gegen verschiedene toxische, aromatische Radikale, die mit der Nahrung ins Blut gelangen, zurückzuführen sein. Diese Vermutung muß allerdings noch experimentell bestätigt werden.

\section{Schlußbemerkung}

Unsere Studie versucht im Rahmen eines standardisierten Testsystems (vor und nach Testmahlzeit) und bei einer ausreichenden Zahl von Neurodermitis-Patienten die immunbiologische Relevanz der Nahrungseinnahme in der Pathogenese der akuten Dermatitis zu dokumentieren.

Unsere Ergebnisse zeigen, daß schon in nüchternem $\mathrm{Zu}$ stand deutlich höhere Werte der ZIK verschiedener IgKlassen, der $\mathrm{C}_{3}$-Aktivator und der akuten phase Proteine auftreten. Die Einnahme einer allergenreichen Testmahlzeit'spiegelte sich wider bei einem erheblichen Teil unserer'Patienten mit einer signifikanten Zunahme dieser $\mathrm{Pa}$ rameter; eine Tatsache, die zu einer direkten Verschlimmerung der klinischen Symptome beitragen kann.

Die o.g. immunbiologischen Merkmale können weiterhin neben Darmdurchlässigkeitsmessungen, Histamin- und 

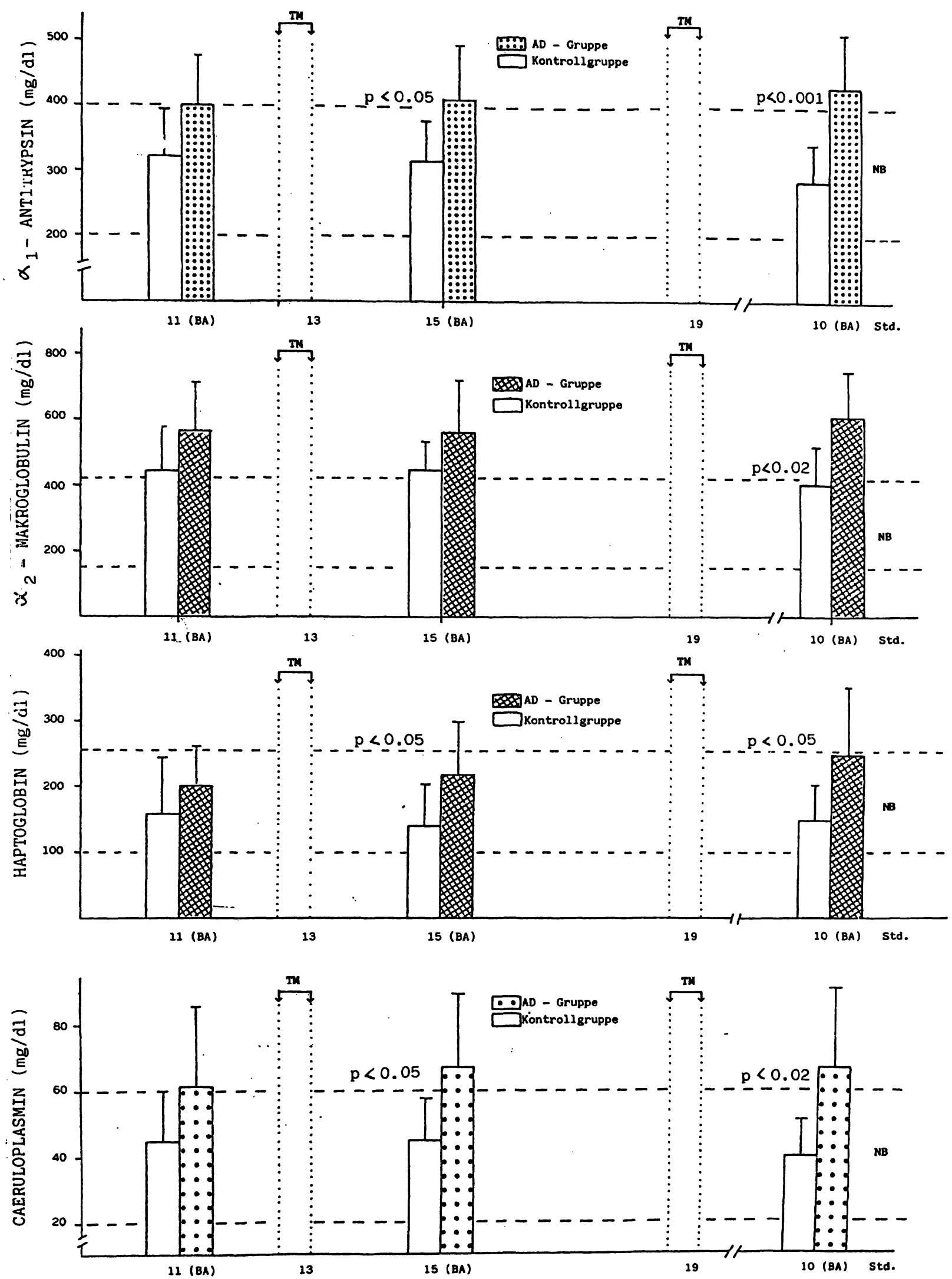

RIU: Radile Immundifusion AD: Atopische Dermatitis IM: Testmahlzeit BA: Blutabnahme NB: Normberelch

Abb. 3: RID.Bestimmung der akute phase Proteine im Serum von Neurodermitikern und Kontrollpersonen vor und nach standardisierten Testmahlzeiten 
RAST-Bestimmungen nach Testmahlzeit (8) als zuverlässiger Marker des Therapieerfolges dienen.

Ein allergenarmer Diätplan, kombiniert mit der Sanierung mikrobieller Herde und mit stoffwechselunterstützenden Maßnahmen, stellen nach unseren Erfahrungen die wichtigsten therapeutischen Eingriffe in die Behandlung der atopischen Dermatitis dar.

Wir danken dem Institut für Klinische Immunologie und Rheumatologie der Universität Erlangen/Nürnberg sowie der Fa. Beh. ringwerke AG. Marburg, und der Fa. Deutsche Pharmacia, Freiburg, für die großzügige Unterstützung dieser Studie.

\section{Schrifttum}

1. STIFLER, W. C.. SEDLIS, E.: Some challenge studies with foods. J. Pediatr. 66. 235 (1965)

2. BOCK, S. A.: The natural history of food sensitivity. J. Allergy Clin. Immunol. 69, 173 (1982)

3. SAMPSON, H. A.: Role of immediate food hypersensitivity in the pathogenesis of atopic dermatitis. J. Allergy, Clin. Immunol. 71,473 (1983)

4. WRAITH D G MERRETT, J ROTH, A. YMAN, L, MERRETT, T. G.: Recognition of food-allergic patients and their allergens by RAST technique and clinical investigaof food-allergic patients and the
tion. Clin. Allergy 9,25 (1979).

tion. Clin. Allergy 9, 25 (1979).
5. GREEN, A. R., VERMA, S., KALRA, V., HADDAD, Z. H.: Evaluation of early-onset 5. GREEN. A. R., VERMA, S., KALRA, V., HADDAD, Z. H.: Evaluation of early-onse
vs. delayed-onset food allergy with RAST. J. Allergy Clin. Immunol. 63, 198 (1979) 6. BROSTOFF, J., CARINI, C., WRAITH. D. G., JOHNS, P.: Production of lgE complexes by allergen challenge in atopic patients and the effect of sodium cromoglycate. Lancet i, 1268 (1979)

7. PAGANEL ( R . IEVINSKI, R. J., BROSTOFF, J., WRAITH, D. C. Immune complexes containing food proteins in normal and atopic subjects after oral challenge and effect of sodium cromoglycate on antigen absorption. Lancet, i, 1270 (1979).
8. IONESCU, G., RADOVICI, D., NEGOESCU, A., PREDA, I., MAHAL, H.: Zirkulierende Immunkomplexe, spezifische IgE gegen Nahrungsmittel- und Inhalationsallergone. Serumhistaminspiegel und Darmpermeabilitätsstorungen bei Neurodermitikern vor und nach Testmahlzeiten. Immun. Infekt. 13, 147 (1985).

9. HANIFIN, J. M., LOBITZ, W. C. : Newer concepts of atopic dermatitis. Arch. Dermatol. 113, 663 (1977).

10. KRAPF, F., RENGER, D., SCHEDEL, I., LEIENDECKER, K., LEYSSENS, H., DEICHER, H.: A PEG-precipitation laser nephelometer technique for detection and characterization of circulating immune complexes in human sera. J. Immunol. Methods 54, 107 (1982).

11. MANCINI G CARBONARA A O HEREMANS J P.ImmunOchemical quantitation of antigens by single radial immunodiffusion. Immunochemistry 2,235 (1965). 12. JACKSON. P. G., LESSAF. H. M., BAKTER, R. W., FERRETT. J., MaCDONALD. D. M.: Intestinal permeability in patients with eczema and food allergy. Lancet, i, 1285 (1981).

13. ISHIZAKA, T., SIAN, C. M., ISHIZAKA, K.: Complement fixation by aggregated IgE through alternative pathway. J. Immunol. 108, 848 (1972).

14. KAZATZHKINE, M. D.. NYDEGGER. V. E.: The human alternative complement pathway. Biology and immunopathology of activation and regulation. Prog. Allergy 30, 193 (1982).

15. KRAPF, F., KALDEN, J. R.: Immunkomplexe und ihre klinische Zuordnung. Laboratoriumsblätter 33,39 (1983).

16. COCHRANE, C. G., KÖFFLER, D.: Immune complex diseases in experimental animals and man. Adv. Immunol. 16, 185 (1973).

17. THEOFILOPOULOS, A. N., DIXON, F. J.: The biology and detection of immune complexes. Adv. Immunol. 28, 89 (1979).

18. MOWAT, A. G., BAUM, J.: Chemotaxis of polymorphonuclear leucocytes from patients with rheumatoid arthritis. J. Clin. Invest. 50, 2541 (1971).

19. MORETTA, L., MINGARI, M. C., ROMANZI, C. A.: Loss of Fc receptors for IgG from human $T$ lymphocytes exposed to $\mathrm{IgG}$ immune complexes. Nature 272, 618 (1978).

20. IONESCU, G., RADOVICI, D. NEGOESCU, A., PREDA. I., KÜRTHI, E., ITZE, W., ITZE, L.: Evaluation of cell-mediated immunity in superinfected atopic eczema by a standardized multipuncture skin test. Infection (in press).

21. KAUFMANN, H. S., FRICK, O. L., FINK, D.: Serum complement $\left(\beta_{1 c}\right)$ in young children with atopic dermatitis. J. Allergy 42, 1 (1968).

22. IONESCU, G.: Biologische Merkmale in der Pathogenese des chronischen Lupus Erythematodes Discoides. Dissertation, Universität des Saarlandes, Saarbrücken (1983)

23. CLEVE, H., ALEXANDER, K., MITZKAT, H. J., NISSEN, P., SALZMANN, I.: Serumglykoproteine bei Diabetes mellitus; quantitative immunologische Bestimmung von saurem $\alpha_{1}$-Glykoprotein, Gc, $\alpha_{2}$-Makroglobulin und Hämopexin bei Diabetikern mit und ohne Angiopathien. Diabetologia 4, 48 (1968).

24. PODLESKI, W. K.: Cytodestructive mechanisms provoked by food antigens. I. Direct, allergic autocytotoxicity. Allergy 40, 157 (1985).

25. DORMANDY, T. L.: Free radical oxidation and antioxidants. Lancet, i, 647 (1978).

Anschrift der Verfasser:

Dr. G. lonescu

Vital-Klinik

Streuweg 100

8755 Alzenau-Michelbach 

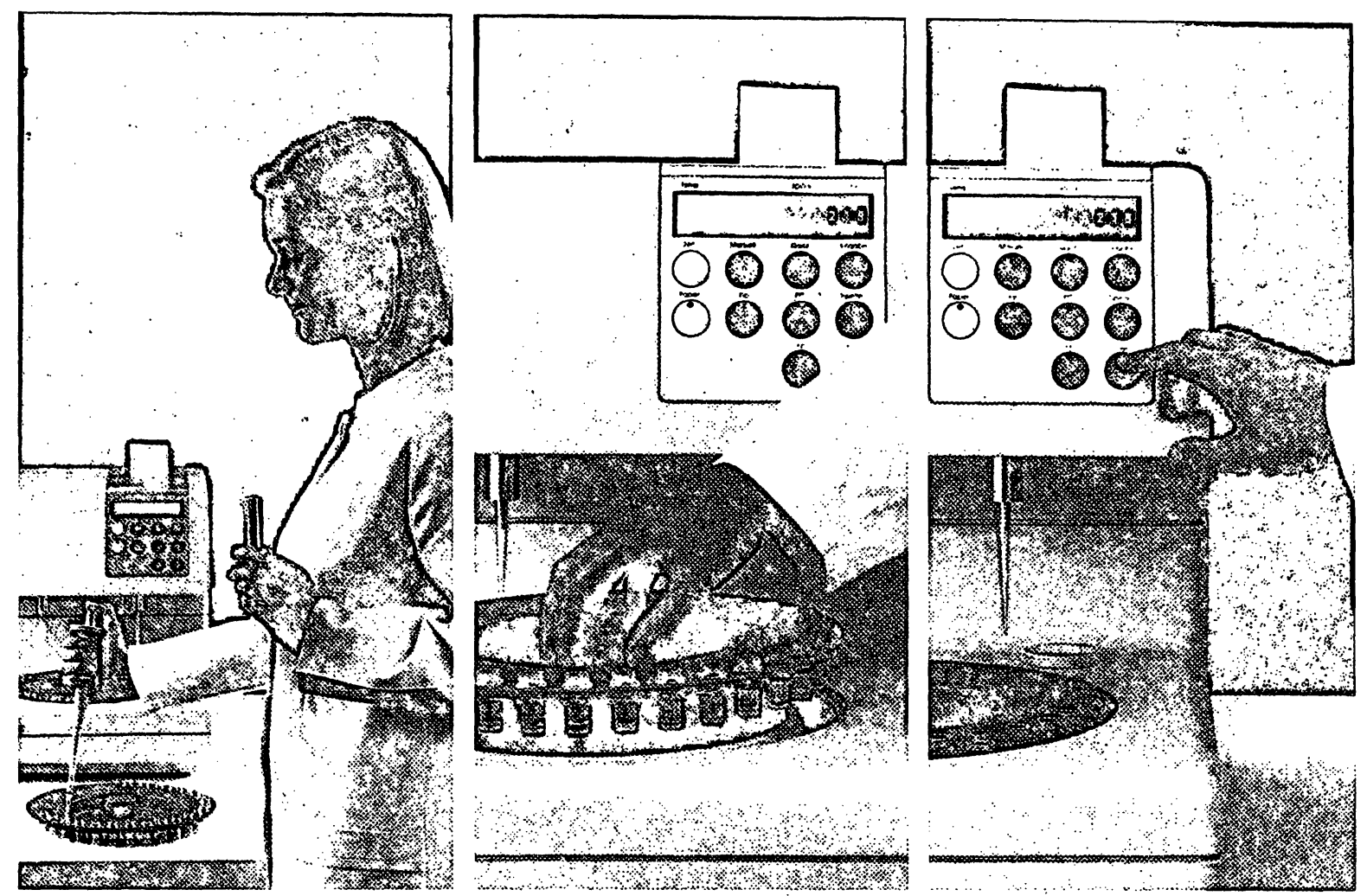

\section{Plasma vorlegen, Probenteller einsetzen, Gerät starten.}

Alles andere macht das Gerät vollautomatisch. So einfach ist Blutgerinnungs-Messung mit dem Coatron von LRE Medizintechnik.

Das Coatron ist ein Coagulometer zur Bestimmung von Fibrinogen. PT (Quick). PTT. TZ und Faktoren. Es überzeugt durch seine hochentwickelte Technik und die fortschrittliche Elektronik made in Germany. - Das Prinzip der optischen Meßtechnik bestimmt die Trübungsänderung auf fotometrischem Weg. Sobald die Gerinnung eingetreten ist, druckt der integrierte Mikroprozessor innerhalb Sekunden die Gerinnungszeit aus. Die Coatron-Technik gewährleistet optimale Reproduzierbarkeit der Meßergebnisse und eine genauere Meßauflösung. Reinigen und Justieren gibt es nicht mehr. Probenteller einsetzen, pipettieren. Gerät starten - fertig. Ale weiteren Arbeiten übernimmt die Geräte-Automatik. - Das Coatron ist nicht nur führend in der Technik. Es wurde auch für sein gutes Design aus- gezeichnet. - Blutgerinnungsmessung mit LRE bedeutet: Der Arbeitsaufwand bei der Gerinnungsbestimmung wird fühlbar reduziert. Fehler werden weitgehend vermieden, die MTA hat mehr Zeit für anspruchsvollere Arbeiten. Coatron. die effektive und leistungsstarke Hilfe für das rationell durchorganisierte Labor.<smiles></smiles>

Wir stellen Geräte zur Probe auf. Sind Sie daran interessiert? Schreiben Sie. rufen Sie uns einfach an oder wenden Sie sich an Ihren Labor-Fachhandel. Als Finanzierungsalternative bieten wir auch den Leasingvertrag an.

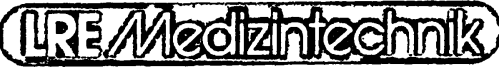

Bausteine für das wirtschaftliche Labor

Postfach 370266

8000 München 37

Tel.: (D 89) 523020 
Isolierung und Identifizierung pathogener Mikroorganismen sind die Voraussetzungen für Diagnose, Therapie, Verhütung von Infektionen und zur Infektionskontrolle.

In dem vorliegenden Buch werden die bisher in jedem qualifizierten mikrobiologischen Labor eingeführten kulturellen und biochemischen Verfahren beschrieben.

Die wichtigsten Daten von ca. 400 als Krankheitserreger geltenden oder aus differentialdiagnostischen Gründen im Bereich der Humanmedizin interessierenden

Bakterienspezies sind in einem kompakten Abriß zusammengefaßt.

Der erste Teil des Buches informiert über Gewinnung, Transport und Verarbeitung von Untersuchungsmaterialien, der Hauptteil enthält sehr ausführlich kommentierte Tabellen zur Identifizierung der Mikroorganismen, und im Anschluß daran werden die im Text erwähnten Methoden unter Angabe von Bezugsquellen für notwendige Hilfsmittel erläutert.

Das zum Gebrauch am Arbeitsplatz bestimmte Buch wendet sich an Mikrobiologen, Hygieniker, Pharmazeuten, medizinisch-technische Assistentinnen und alle diejenigen, die routinemäßig bakteriologische Untersuchungen durchführen oder sich im Praktikum auf diese Tätigkeit vorbereiten.

$--8<-$

\section{An Verlag Kirchheim, Kaiserstraße 41, 6500 Mainz}

Ich bestelle gegen Rechnung ......... Expl. Bürger/Hussain:

Tabellen und Methoden zur med.-bakteriologischen Laborpraxis, zum Preis von DM 68,-

Name/Praxis:

Straße:

PLZ: Ort:

Datum: Unterschrift:
Format $17 \times 24 \mathrm{~cm}$, 256 Seiten, Abbildungen,

Tabellen, PVC-Einband, ISBN 3-87409-006-X, DM 68,-

Bezug über Verlag oder Fachbụchhạndlung

VERLAG KIRCHHEIM MAINZ 